\title{
Design A Low Pass Fir Filter By Using Blackman Harris Kaiser And Tukey Window Techniques
}

\author{
Manju Kanwar', Ruchi Baghel ${ }^{2}$, Pranay Kumar Rahi ${ }^{3}$ \\ B.E. Scholar, Department of EEE, Institute of Technology Korba Chhattisgarh India ${ }^{1,2}$ \\ Assistant Professor, Institute of Technology Korba Chhattisgarh India ${ }^{3}$
}

\begin{abstract}
The input signal has a great influence on the performance of the system in digital control system. Processing of input signals is important to get useful signal in has processing of digital signal finite impulse response (fir) filter plays on important role. The aim of this paper is digital FIR filter designed by different window techniques perform better for reducing noise various window method such as Blackman window, Kaiser window, tucky window. We found that filter designing by window method is easy and fast.
\end{abstract}

Keywords: DSP, Digital Filter, Low Pass Filter, and Blackman Harris Window, Kaiser window and tukey window.

\section{INTRODUCTION}

\subsection{DIGITAL SIGNAL PROCESSING (DSP)}

Digital signal processing techniques play a major role in our life. Digital signal processing techniques are widely used in biomedical field to facilitate the monitoring, diagnosis and analysis of abnormalities in the body. It is said that the origin of DSP technique can be traced to the seventeenth century when finite difference methods numerical integration methods and numerical interpolation methods were developed to solve physical problem involving continuous variables and function[6].

\section{APPLICATION OF DSP}

Some selected application of digital signal processing that are often encountered in daily life are listed as follows;

1. Military Radar signal processing, sonar signal processing, navigation etc.

2. Medicine Medical diagnostic instrumentation such as computerised tomography(CT), X-ray scanning, magnetic resonance imaging etc.

3 .Signal filtering Removal of unwanted background noise, removal of interference, separation of frequency bands and shaping of the signal spectrum[6].

\subsection{Digital filter}

It is a discrete system which can do a series of mathematic processing to the input signal, due to which we can obtain the desired Information from the input signal the transfer function for a linear time invariant digital filter is usually expressed as:

$$
G(z)=\frac{\sum_{j=0}^{M} b_{j} z^{-j}}{1+\sum_{i=0}^{N} a_{i} z^{-i}}
$$

Where $a_{i}$ and $b_{j}$ are coefficients of the filter in $Z$ transform[4]. There are different kinds of digital filters, and also many different ways to classify them. Based on its function, the FIR filters can be categorized into four categories;

a) Low pass filter

b) High pass filter

c) Band pass filter

d) Band stop filter

The following are some steps for designing a digital filter: 1) According to the given requirements make sure of the property of a digital filter.

2) Use a discrete LTI system function to approach to the properties.

3) To design the system function Make use of algorithms.

4) To achieve the task use a computer simulation or hardware.

In different areas digital filter, the input and output signals are digital or discrete time sequence. Digital filters are categorized in two parts as finite impulse response (FIR) and infinite impulse response[7].

\subsection{FIR filter}

FIR filter are particularly useful for application where exact linear phase response is required. The FIR filter is generally implemented in a non-recursive way which guarantees a stable filter FIR filter design essentially consist of two parts:

1.Approximation problem

2.Realization problem

There are essentially three well-known method for FIR filter design namely:

1.The window method

2.The frequency sampling technique 
3.Optimal filter design methods[9].

\subsection{IIR filter}

Infinite impulse response (IIR) filter has a feedback loop and is recursive structure, and it. IIR filter are not linear phase and the precision of amplitude frequency characteristic is very high[6].

\subsection{FIR filter have the following advantages over IIR filter}

1) They can have an exact linear phase.

2) They are always stable.

3) The design methods are generally linear.

4) They can be realised efficiently in hardware.

5) The filter start-up transients have finite duration.

\subsection{Comparison of FIR and IIR}

1) The phase of the IIR filter is not linear while the FIR filter the phase is strictly linear.

2) In FIR filter finite precision arithmetic error is very small and has a non-recursive structure. While IIR filter parasitic oscillation may occur in the operation of IIR filter and has a recursive structure.

3) Fast Fourier Transformation cannot be used in IIR but can be used in FIR filter.

\section{BRIEF INTRODUCTION TO MATLAB}

\subsection{MATLAB}

MATLAB stands for matrix laboratory. It is a technical computing environment for high performance numeric computation and visualisation. It integrates numerical analysis, matrix computation, signal processing and graphics is an easy-to-use environment where problems and solution are expressed just as they are written mathematically, without traditional programming. MATLAB allows us to express the entire algorithm in a few dozen lines, to compute the solution with great accuracy in a few minutes on a computer, and to readily manipulate a three-dimensional display of the result in colour[1]. MATLAB provides a complete solution for engineering design, scientific research, and many other scientific fields. It shows the advanced level in international scientific computing software world[2].

\subsection{Advantages of MATLAB}

1) Easy-to-use programming language

2) Powerful image processing capabilities

3) Collection of modules Toolbox wide range of application

4) Data processing power is very strong
5) Developing the user interface

6) The program interface is very practical and publishing platform[2].

\section{WINDOW TECHNIQUE}

In this method, a certain bandwidth is generated using a truncated ideal low-pass filter, and then we use a selected window to get certain stop-band attenuation. The filter length $\mathrm{L}$ can be changed in the transition band to meet a specified roll-off rate. We start with windowed, truncated low-pass filters, then do it for other kind of filters, like band-stop. Band-pass, and high-pass filters can also be achieved by several techniques.

Any finite-length of a low-pass impulse response may be considered as the product of a window function $\mathrm{W}$ and the infinite-length low-pass impulse response.

$$
\mathrm{a}=\frac{\sin (\boldsymbol{\omega}[\boldsymbol{n}-M])}{\pi[n-M]} \mathrm{W}_{\mathrm{L}}[\mathrm{n}-\mathrm{M}]
$$

Here are some of the basic information on standard window.

\subsection{BLACKMAN HARRIS}

The Blackman Harris window function can be written as;

$$
W(n)=\left\{\begin{array}{cc}
0.42-0.5 \cos \left(\frac{2 \pi n}{N}\right)+0.08 \cos \left(\frac{4 \pi n}{N}\right), & 0 \leq \\
0 & \text { elsewhere }
\end{array}\right.
$$$$
N \leq n-1
$$

\subsection{KAISER WINDOW}

The Kaiser window function can be written as;

$W(n)=\left\{\begin{array}{cc}I_{0}(\beta \sqrt{1-(2(n+1)(N+1),}, & 0 \leq N \leq n-1 \\ 0 & \text { elsewhere }\end{array}\right.$

\subsection{TUKEY WINDOW}

The tukey window function can be written as;

$\mathrm{W} \tau(n)=$

$$
\left\{\begin{array}{c}
\frac{1\left[1+\cos \left(\pi\left(\frac{2 n}{\alpha(M-1)}-1\right)\right)\right], 0 \leq n \leq \frac{\alpha(M-1)}{2}}{2} \\
1, \frac{\alpha(M-1)}{2} \leq n \leq(M-1)\left(1-\frac{\alpha}{2}\right) \\
\frac{1}{2}\left[1+\cos \left(\pi\left(\frac{2 n}{\alpha(M-1)}-\frac{2}{\alpha}+1\right)\right)\right],(M-1)\left(1-\frac{\alpha}{2}\right) \leq n \leq(M-1)
\end{array}\right.
$$

\section{SIMULATION AND RESULT}

Table - 1. Parameter specification

\begin{tabular}{|c|c|}
\hline PARAMETER & VALUES \\
\hline Sampling Frequency $\left(\mathrm{F}_{\mathrm{s}}\right)$ & 48000 \\
\hline Cut-off Frequency $\left(\mathrm{F}_{\mathrm{c}}\right)$ & 10800 \\
\hline Order $(\mathrm{N})$ & 15 \\
\hline
\end{tabular}


onse of blackman-harris

Table -2 . Frequency and magnitude.

\begin{tabular}{|l|l|l|l|}
\hline frequency & $\begin{array}{l}\text { Blackman- } \\
\text { harris }\end{array}$ & Kaiser & tukey \\
\hline $0.1 \pi$ & -0.042 & 0.126 & -0.074 \\
\hline $0.2 \pi$ & -0.101 & 0.257 & -0.104 \\
\hline $0.3 \pi$ & -0.068 & 0.180 & -0.052 \\
\hline $0.4 \pi$ & 0.125 & -0.027 & 0.114 \\
\hline $0.5 \pi$ & 0.419 & -0.153 & 0.458 \\
\hline $0.6 \pi$ & 0.675 & 0.027 & 0.698 \\
\hline $0.7 \pi$ & 0.619 & 0.565 & 0.621 \\
\hline $0.8 \pi$ & 0.085 & 0.667 & 0.004 \\
\hline $0.9 \pi$ & -1.654 & -0.520 & -1.270 \\
\hline$\pi$ & -3.593 & -2.798 & -3.636 \\
\hline
\end{tabular}

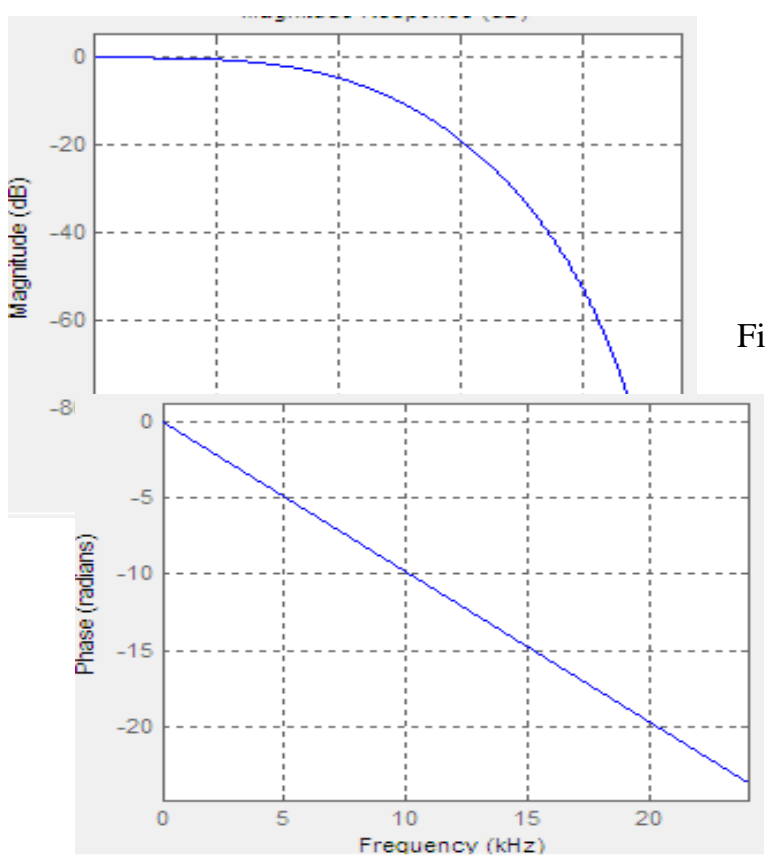

re-1. Magnitude response of blackman-harris

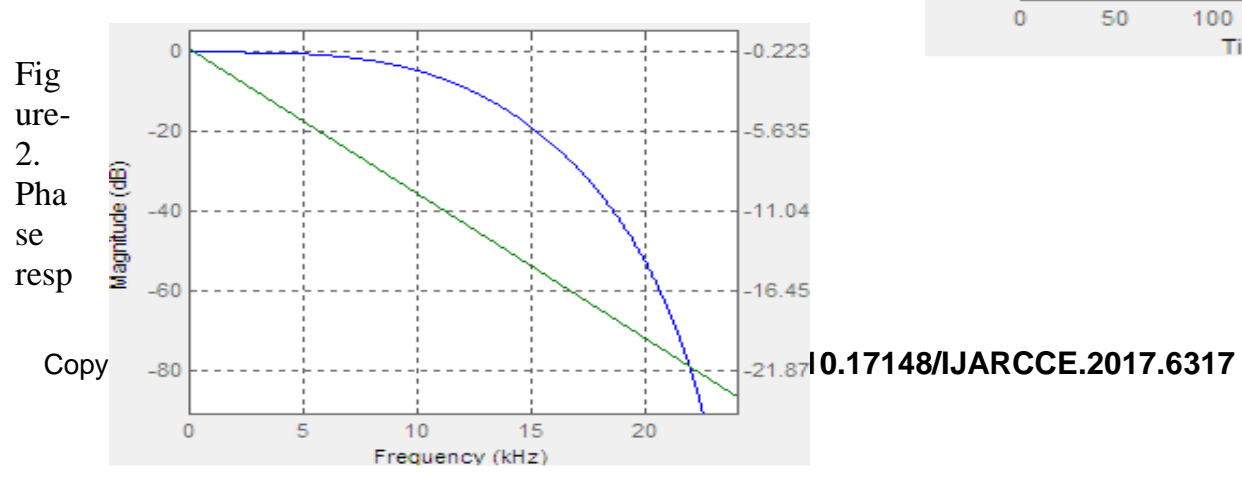

Figure-3. Magnitude and phase response of blackmanharris

Figu
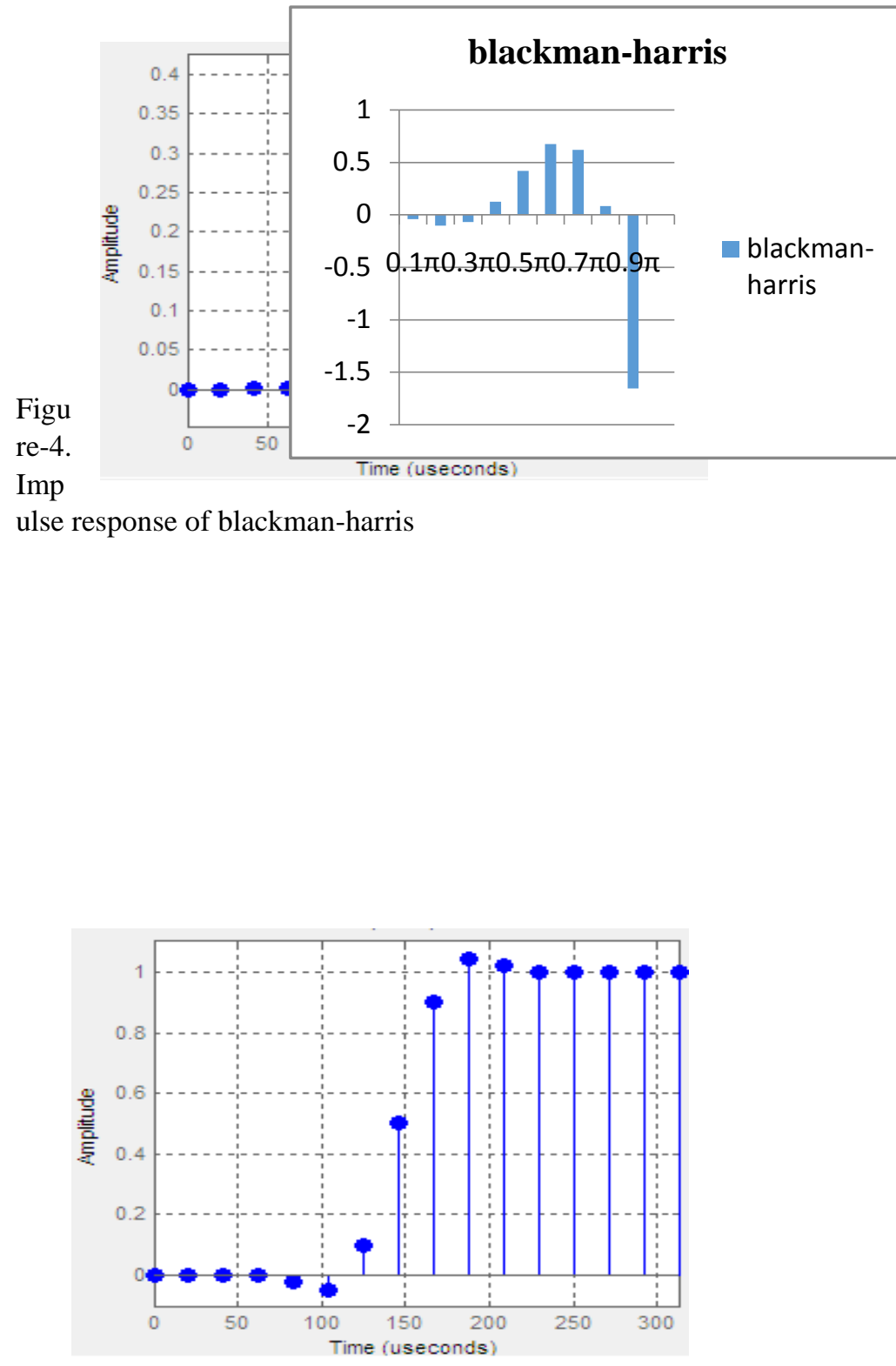
Figure-7. Filter coefficient of blackman-harris

Figure-5. Step response of blackman-harris

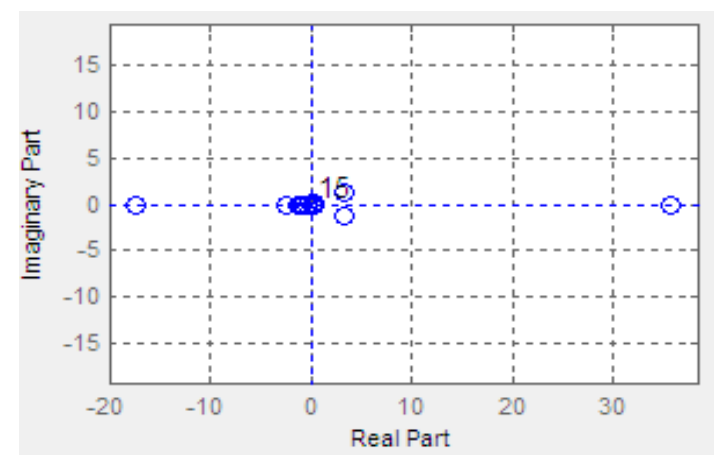

Figure-6. Poles and zeros plot of blackman-harris

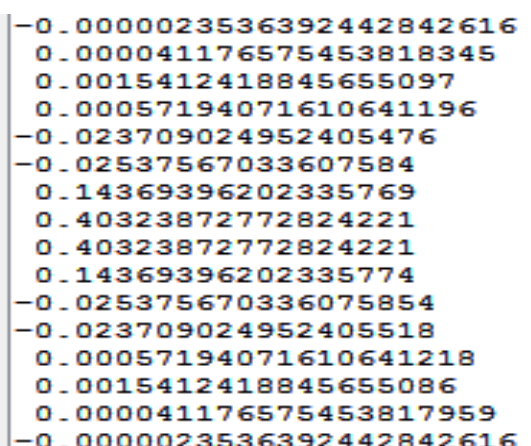

Figure-8. Frequency and magnitude plot of blackmanharris
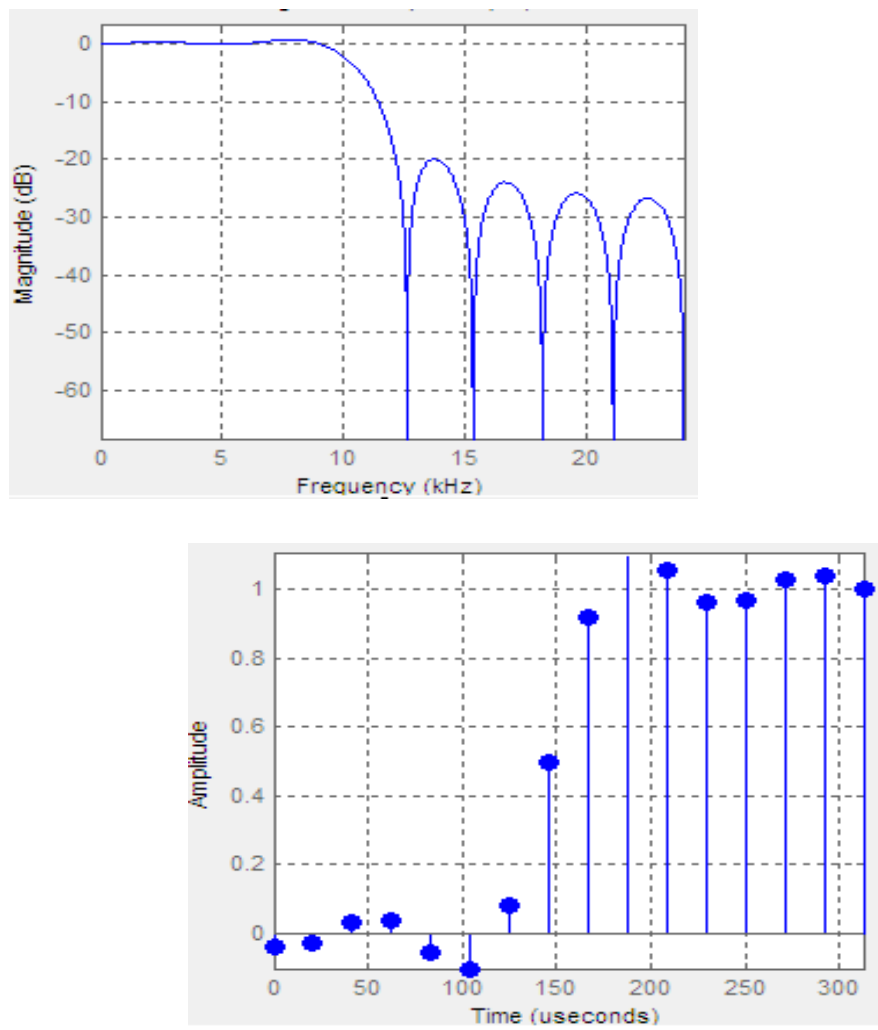

Figure-9. Magnitude response of Kaiser window 


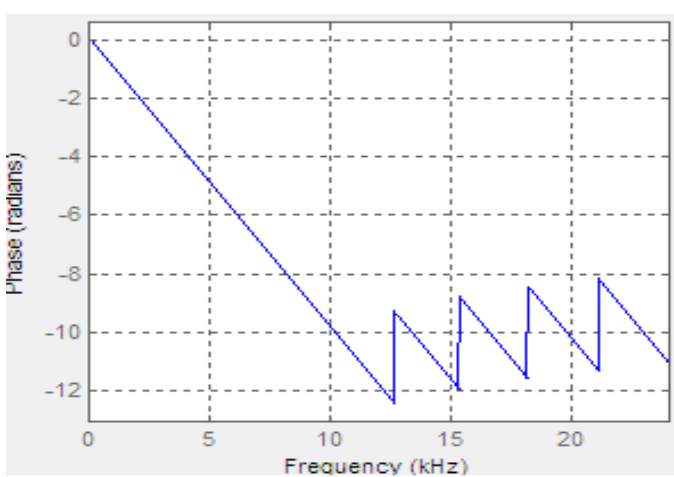

window
Figure-10.

phase response of Kaiser window

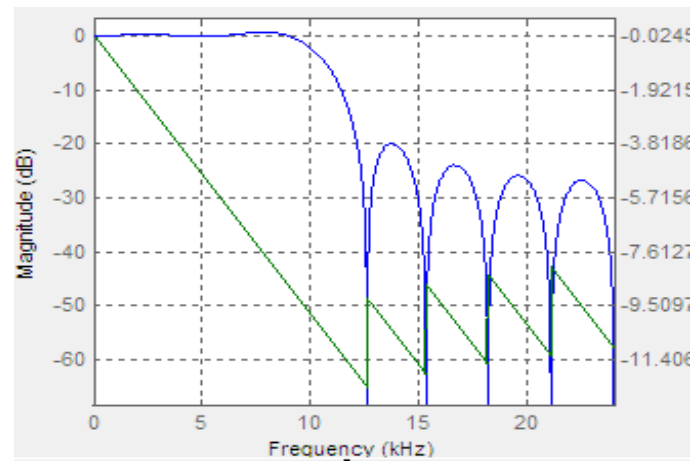

Figure-11. magnitude and phase response of Kaiser

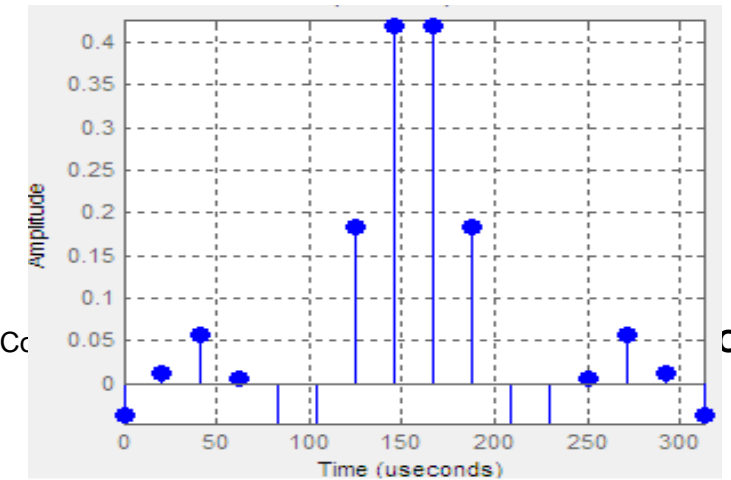


Figure-15. filter coefficient of Kaiser window

Figure-16. Magnitude and frequency plot of kaiser window

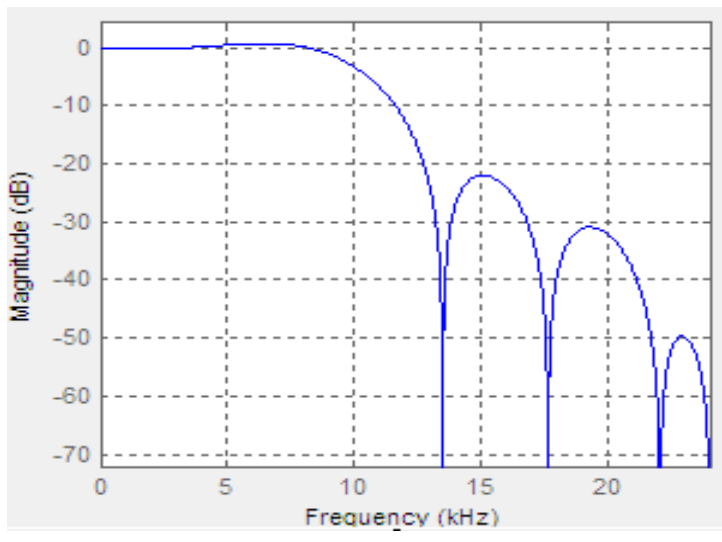

Figure-17. Magnitude response of tukey window

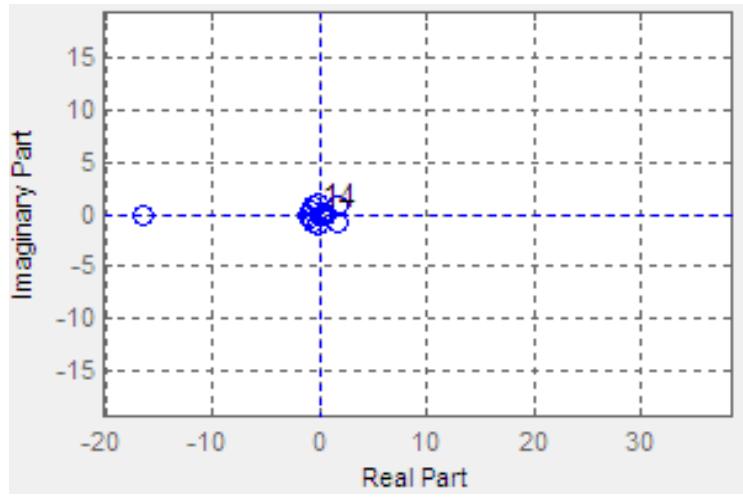

Figure-

18.Phase response of tukey window

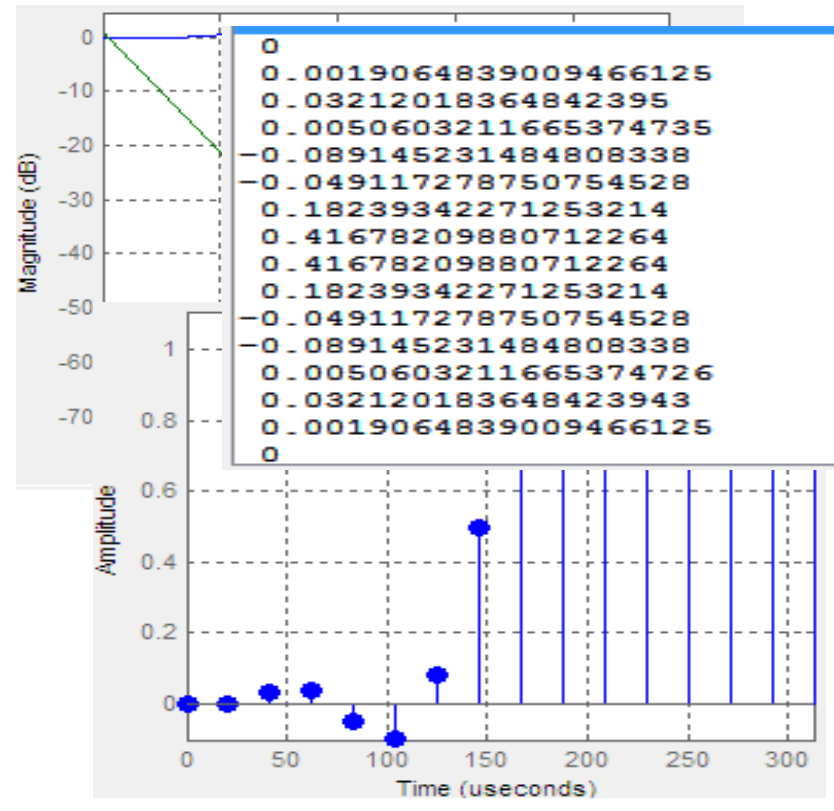

Figure-19. Magnitude and phase response of tukey window

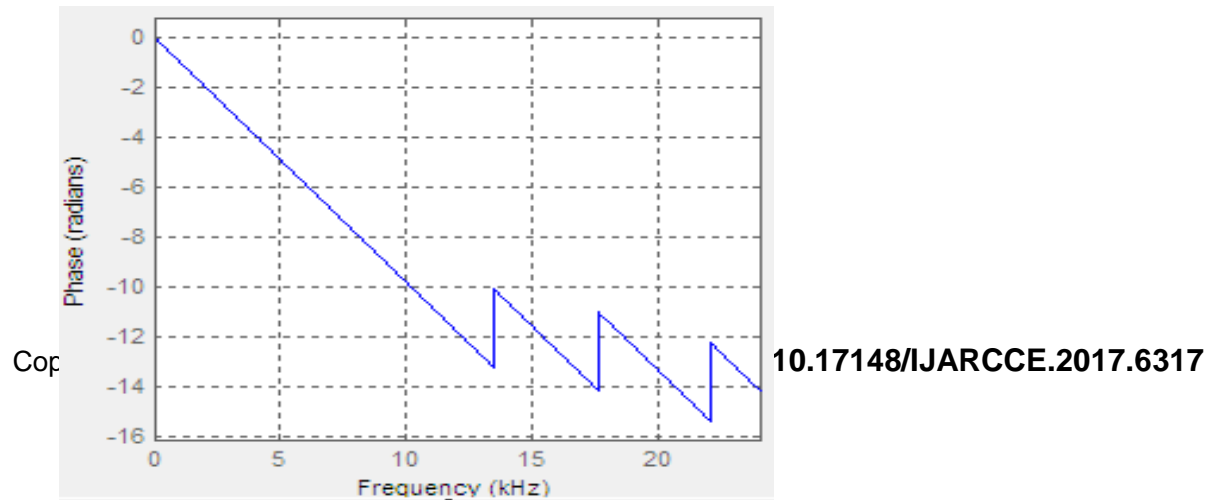




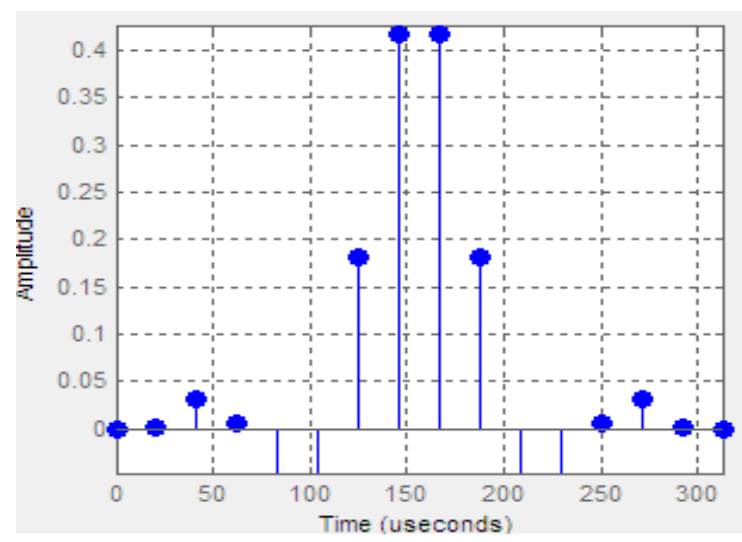

Figure-20. Impulse response of tukey window

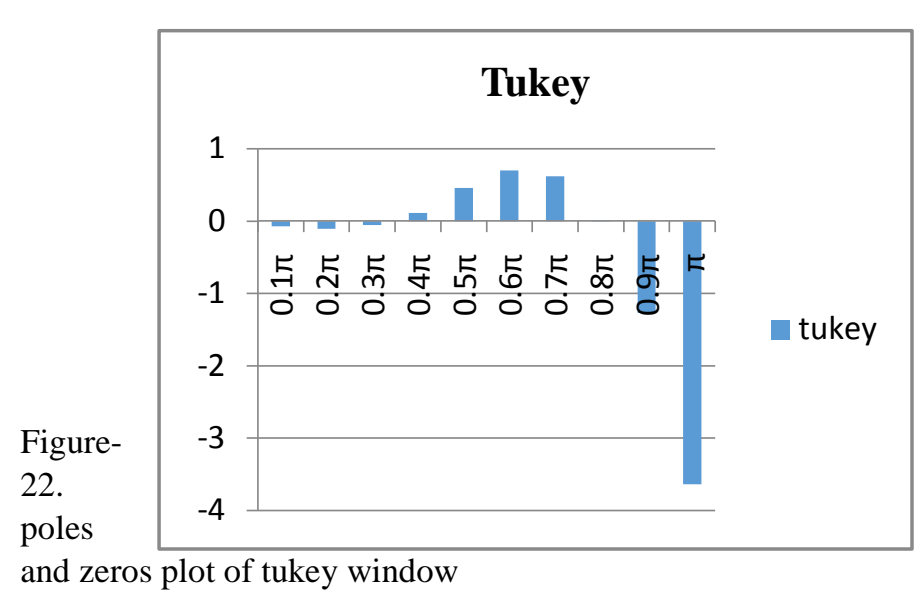

Figure-23. Filter coefficient of tukey window

Figure-24. Frequency and magnitude plot of tukey window

Figure-21. Step response of tukey window 


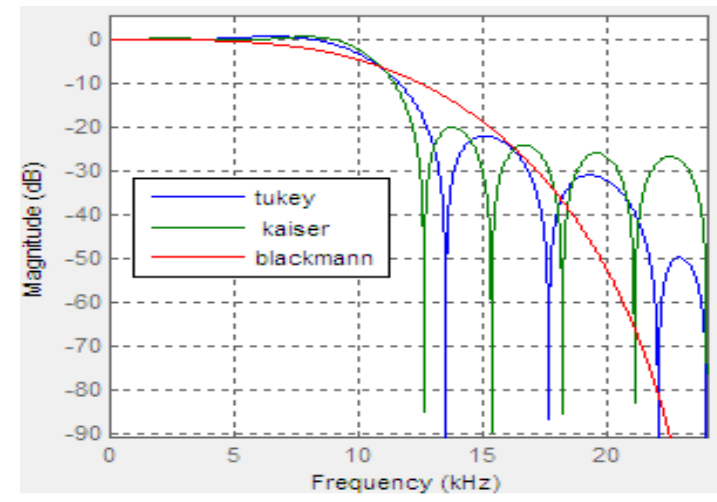

Figure-

26.

phase compar ison of tukey, Kaiser and blackm an

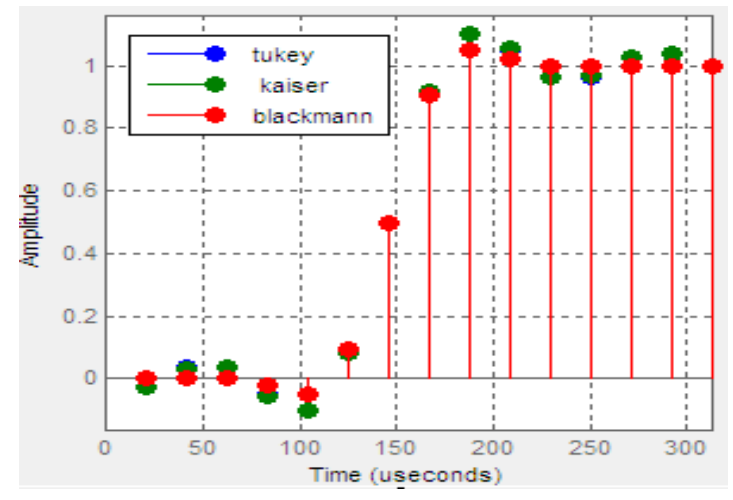

Figure-25. Magnitude comparison of tukey,Kaiser and blackman

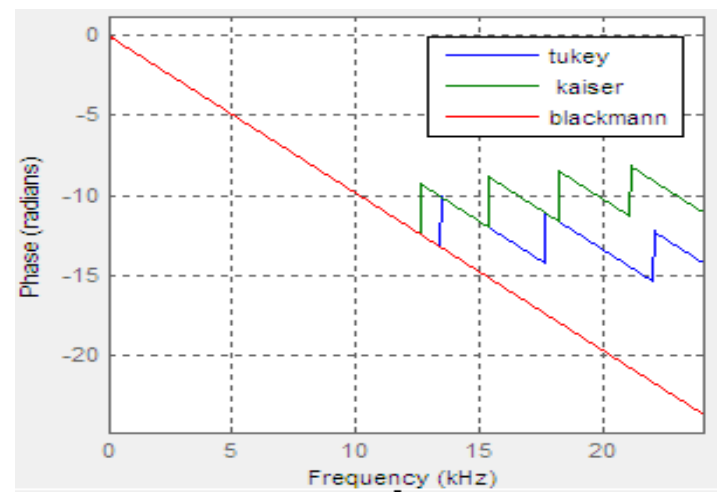

Figure-27. magnitude and phase comparison of tukey, kaiser and blackman-harris

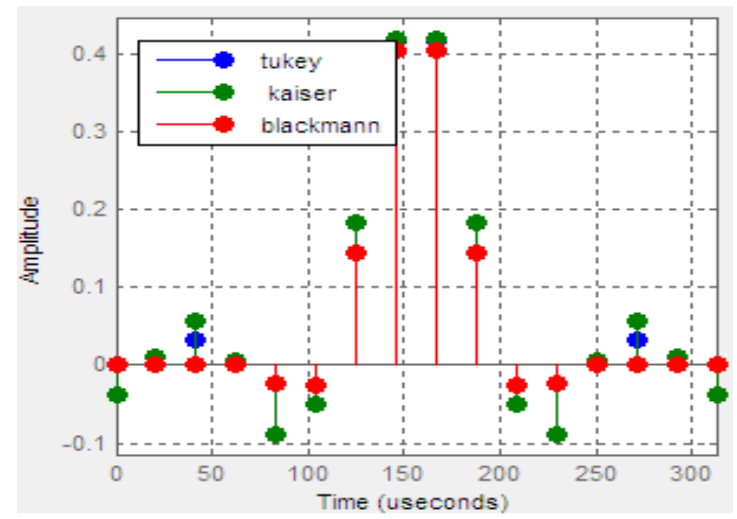

Figure-28. Impulse response comparison of tukey, kaiser and blackman-harris

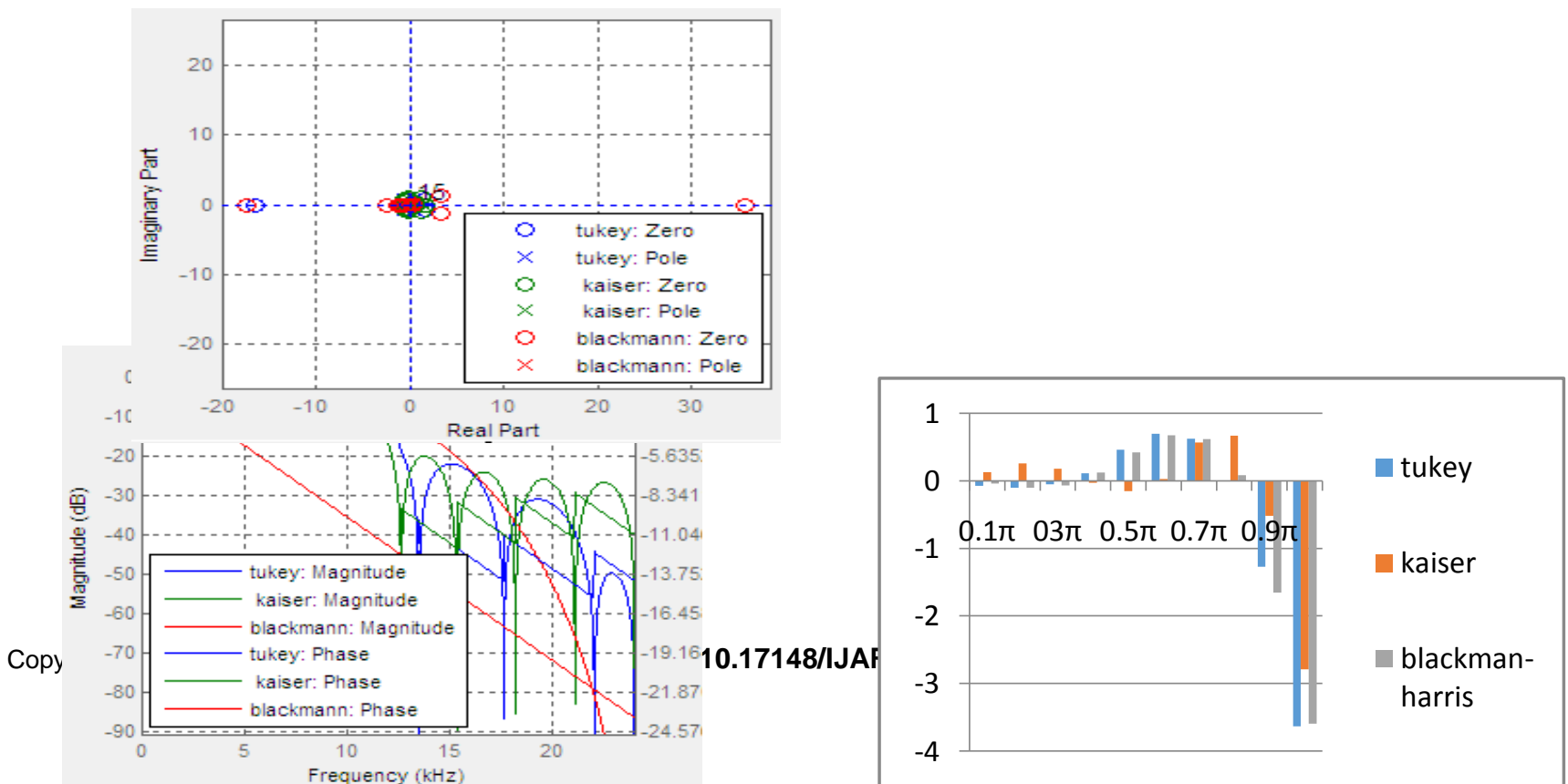


Figure-29. Step comparison of tukey, kaiser and blackman-harris

Figure-30. Poles and zeros comparison of tukey, kaiser and blackman-harris

Figure-31. Frequency and magnitude plot comparison of tukey, kaiser and blackman-harris

\section{CONCLUSIONS}

Window function is used to design different filters like band-pass, low-pass and multi-pass-band filter. Different windows were selected for filter. When windows were applied on the signal they filtered that frequencies which were not in its range and kept that frequencies which were in it range. So if we want to pass or stop some frequencies window function can used for it. In low pass filter only low frequencies were pass and other were filtered out. Band-pass pass a portion of frequencies and other were filters out. In short window function can be applied to any signal using any type of filter to get desired frequencies

\section{REFERENCES}

[1]. Sanjit K. Mitra "digital signal processing" a computer based Approach $2^{\text {nd }}$ Edition McGraw Hill, pp.1.

[2] DSP for MATLAB and LabVIEW. Forester W. Isen.

[3] Digital Signal Processing John G. Prokis. Dimitris G. Manolakis Fourth Edition. 2007.

[4] Signal Processing First James H. McClellan. Ronald W. Schafer. Mark A. Yoder. 2003

[5]. Michael Weeks, "digital signal processing" Using MATLAB and Wavelets, 2007 edition, Infinity Science Press, pp. 1

[6]. S Salivahanan, C Gnanapriya, A Vallavaraj, "digital signal processing" Tata McGraw-Hill, $2^{\text {nd }}$ Edition pp.3.731.

[7]. Richars G. lyons, Understanding digital signal processing" PEARSON, , $2^{\text {nd }}$ Edition.

[8]. A Nagoor Kani, “digital signal processing” McGraw-Hill Education, $2^{\text {nd }}$ Edition

[9]. Alan V. Oppenheim, Ronald W. Schafer,"digital signal processing”, PEARSON education, 2015 Edition.

\section{AUTHORS}

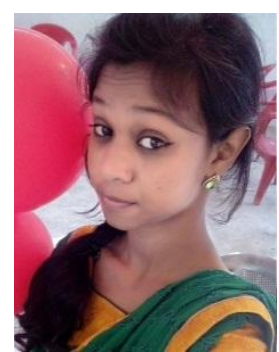

Manju Kanwar persuing bachelor of engineering in electrical and electronics engineering in 6 th semester from institute of technology korba,Chhattisgarh Swami Vivekanand Technical University, India

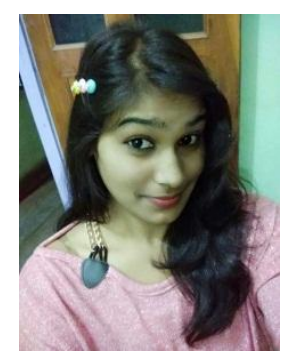

Ruchi Baghel persuing bachelor of engineering in electrical and electronics engineering in 6 th semester from institute of technology korba,Chhattisgarh Swami Vivekanand Technical University, India

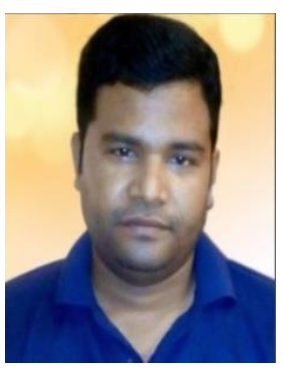

Pranay Kumar Rahi received the bachelor of technology degree in Electronics and Telecommunication engineering collage, guru ghashidas university, bilaspur Chhattisgarh, india in 2004, and persuing master of engineering in electronics and communication engineering from national institute of technical teacher's training \& research, Punjab university, Chandigarh, india. Presently working as assistant professor in department of electrical and electronics engineering, institute of technology, korba Ch.hattisgarh since 2008. He has authored more than 40 research publications and published journal paper and research papers in the leading international and national journal. This primary research interest includes digital signal processing, VLSI design, control systems and digital electronics and logic design. 